\section{(A) Check for updates}

Cite this: Nanoscale, 2020, 12, 15869

\title{
A Henry's law method for generating bulk nanobubbles $\uparrow$
}

\author{
Gianluca Ferraro, Ananda J. Jadhav and Mostafa Barigou (D) *
}

\begin{abstract}
A new technique for generating bulk nanobubble suspensions has been developed based on Henry's law which states that the amount of dissolved gas in a liquid is proportional to its partial pressure above the liquid. This principle which forms the basis of vacuum degasification has been exploited here to produce stable bulk nanobubbles in excess of $10^{9}$ bubble $\mathrm{mL}^{-1}$ in pure water, through successive expansion/compression strokes inside a sealed syringe. We provide evidence that the observed nano-entities must be gas-filled nanobubbles by showing that: (i) they cannot be attributed to organic or inorganic impurities; (ii) they disappear gradually over time whilst their mean size remains unchanged; (iii) their number density depends on the concentration of dissolved gas in water and its solubility; and (iv) added sparging of gas enhances process yield. We study the properties of these nanobubbles including the effects of type of dissolved gas, water $\mathrm{pH}$ and the presence of different valence salts on their number density and stability. Given the potential of the technique for large scale production of nanobubble suspensions, we describe a successfully tested automated model and outline the basis for process scale-up.
\end{abstract}

Received 28th April 2020, Accepted 8th July 2020

DOI: 10.1039/d0nr03332d rsc.li/nanoscale action between two charged surfaces. Thus, it would seem plausible that the surface charge might govern the stability of BNBs, and there is mounting evidence in support of such an argument. ${ }^{10}$ Nonetheless, a complete physical model is still missing.

Bulk nanobubbles have been reported in numerous papers $(>150)$ and several patents. Despite this multitude of scientific reports, the mystery behind the longevity of BNBs is still causing some scepticism about their existence, leading to speculation and controversy about the nature of BNBs and their origin. The small minority of works which dispute the existence of BNBs have tended to attribute them to solvent/oil contamination, solid impurities, mesoscale aggregates or supramolecular structures. ${ }^{12-19}$ Whilst direct evidence is still missing, recently, however, a significant body of indirect corroborative evidence has been reported that BNBs do exist and they are stable in pure water as well as in aqueous organic solvent solutions. ${ }^{1,3,4,10,20-23}$

Whilst research in this area is still in its infancy, already a wide range of potential BNB applications have been suggested or have indeed been industrially implemented, including drag reduction, ${ }^{24}$ enhanced germination rate of seeds, ${ }^{25,26}$ froth flotation, ${ }^{27-31}$ improved engine efficacy using hydrogen nanobubbles, ${ }^{32,33}$ nanobubbles as ultrasound contrast agent, ${ }^{34-39}$ promotion of the physiological activity of living organisms, ${ }^{6}$ sterilisation of bacteria, ${ }^{40}$ surface cleaning, ${ }^{41-43}$ wastewater treatment ${ }^{44}$ and medical applications such as improved blood oxygenation, ${ }^{45}$ therapeutic drug delivery, ${ }^{38,39,46,47}$ reversal of hypoxic conditions, ${ }^{48,49}$ and use in
School of Chemical Engineering, University of Birmingham, Edgbaston, Birmingham B15 2TT, UK. E-mail:m.barigou@bham.ac.uk

$\dagger$ Electronic supplementary information (ESI) available. See DOI: 10.1039/ d0nr03332d 
diagnostics and gene therapy. ${ }^{50}$ Thus, it appears that there is immense scope for nanobubbles to impact and perhaps revolutionise many current industrial and medical processes.

A number of techniques have been reported for the generation of BNBs including single nanobubble electrolysis, ${ }^{51-55}$ acoustic cavitation, ${ }^{1,3,22}$ hydrodynamic cavitation, ${ }^{56,57}$ fluidic oscillation, ${ }^{58}$ nano-membrane filtration, ${ }^{59}$ water-solvent mixing, ${ }^{3,21,60,61}$ laser, $^{62,63}$ periodic pressure changes, ${ }^{23}$ compression and decompression of gas, ${ }^{64-66}$ and chemical reactions. ${ }^{67}$ Each method has its own advantages and shortcomings. For example, electrochemical and chemical reaction methods are limited to specific gases, for instance, electrolysis of water can only produce hydrogen and oxygen nanobubbles. On the other hand, hydrodynamic cavitation, ultrasound cavitation and membrane filtration are more general and can be utilized with a wide range of gases. These methods, however, if used in an uncontrolled fashion, are prone to contamination, they have low resistance to corrosive chemicals, which restricts the use of reactive gases and solutions, and they tend to be energy intensive. Therefore, to serve industrial and medical needs, the search continues for BNB generation techniques which can produce large concentrations of BNBs, are 'clean', cost effective and amenable to scale-up and process control.

In this paper, we present a new technique based on Henry's law's principle of vacuum degasification, for generating bulk nanobubble suspensions by means of successive expansion/ compression strokes inside a sealed syringe, and provide multiple evidence that the observed nano-entities must be gasfilled nanobubbles. We study the influence of the number of expansion-compression cycles, the type and concentration of dissolved gas in water and its solubility on the formation of BNBs, and we demonstrate how sparging of added gas enhances process yield. We also investigate systematically the effects of water $\mathrm{pH}$ as well as the presence of different valence salts on the number density of BNBs formed and their stability. Finally, we propose a tested automated model of the technique and outline the basis for process scale-up.

\section{Experimental}

\subsection{Materials}

Ultrapure water (Type 1), henceforth referred to as pure water or simply water, from a Millipore purification system (Avidity Science, UK), of electrical conductivity $0.055 \mu \mathrm{S} \mathrm{cm}^{-1}$ and $\mathrm{pH}$ 6.7 at a temperature of $20{ }^{\circ} \mathrm{C}$, was used in all experiments. Sodium chloride $(\mathrm{NaCl}, \geq 99.5 \%)$, calcium chloride $\left(\mathrm{CaCl}_{2}\right.$, $\geq 99 \%)$ and aluminium chloride $\left(\mathrm{AlCl}_{3}, 99.9 \%\right)$ were obtained from Sigma Aldrich. Potassium hydroxide (KOH, 98\%) and hydrochloric acid $(37 \% \mathrm{HCl}$ AR grade) were purchased from VMR Chemicals (UK). Dry air, nitrogen and Argon gases of purity $>99.5 \%$, were supplied by BOC (UK). All solvents and reagents used were of the highest purity grade available on the market. Prior to use, all glassware was cleaned by immersion for $30 \mathrm{~min}$ in a $10 \%$ aqueous solution of potassium hydroxide inside an ultrasound bath, followed by rinsing with copious amounts of ultrapure water, drying in a microwave oven and flushing with a stream of high-purity dry nitrogen gas. Polypropylene plastic syringes used for BNB generation were thoroughly cleaned, and all traces of lubricating silicone oil removed, by immersion for $30 \mathrm{~min}$ in a $10 \%$ aqueous solution of analytical grade ethanol (99.9\% pure, Fisher Scientific, UK), followed by thorough rinsing with ultrapure water and drying with a stream of high-purity dry nitrogen gas. Prior to experimentation, the purified water and all stock solutions were examined using the Nanosight instrument (described further below) employed for the measurement of BNBs, to ascertain that no significant levels of nanoscale impurities could be detected. In all cases, the BNB suspensions formed were stored in $20 \mathrm{~mL}$ air-tight glass vials for further analysis.

\subsection{Henry's law method of bulk nanobubble generation}

According to Henry's law, at constant temperature, the saturation concentration of gas in a given liquid, i.e. the amount of dissolved gas, is directly proportional to the partial pressure of the gas above the liquid, thus:

$$
C=H \times P_{\mathrm{g}}
$$

where, $C$ is the gas solubility at a given temperature in a particular solvent, $P_{\mathrm{g}}$ is the partial pressure of gas and $H$ is Henry's law constant. Therefore, subjecting the liquid to reduced pressure makes the dissolved gas less soluble and, hence, leads to gas molecules being released. This principle forms the basis of the technique of vacuum degasification. ${ }^{68}$

We show that subjecting water to vacuum pressure at constant temperature inside a syringe creates gas undersaturation which, when followed by vacuum release, leads to the formation of BNBs; we call this an expansion-compression method, as schematically illustrated in Fig. 1. Initially, a syringe is filled with pure water and after expelling any air trapped within, the syringe tip is sealed using a Luer lock cap. The water is then depressurised by quickly pulling the syringe plunger out and then repressurised by instant release of the plunger which travels at a relatively high velocity under the action of vacuum pressure (typically $\sim 7 \mathrm{~cm} \mathrm{~s}^{-1}$ ); these two steps represent one full cycle of the BNB generation process. It should be noted that a sufficient amount of vacuum needs to be created inside the syringe in order to cause enough dissolved gas to be released as well as have enough pressure differential during the compression stage to enable the formation of BNBs. In other words, the pressure inside the syringe during the expansion stroke needs to be as low as possible. Many successive cycles are required to produce a sufficiently large number of BNBs. The process is demonstrated in a video provided in ESI. $\dagger$

It should be noted, however, that if the absolute pressure of water is reduced to its vapour pressure at the prevailing temperature, it boils and vapour bubbles develop, a process commonly known as cavitation. The vast majority of our experiments were conducted at a temperature of $20{ }^{\circ} \mathrm{C}$ with a minimum absolute pressure of $\sim 0.048$ atm inside the water- 


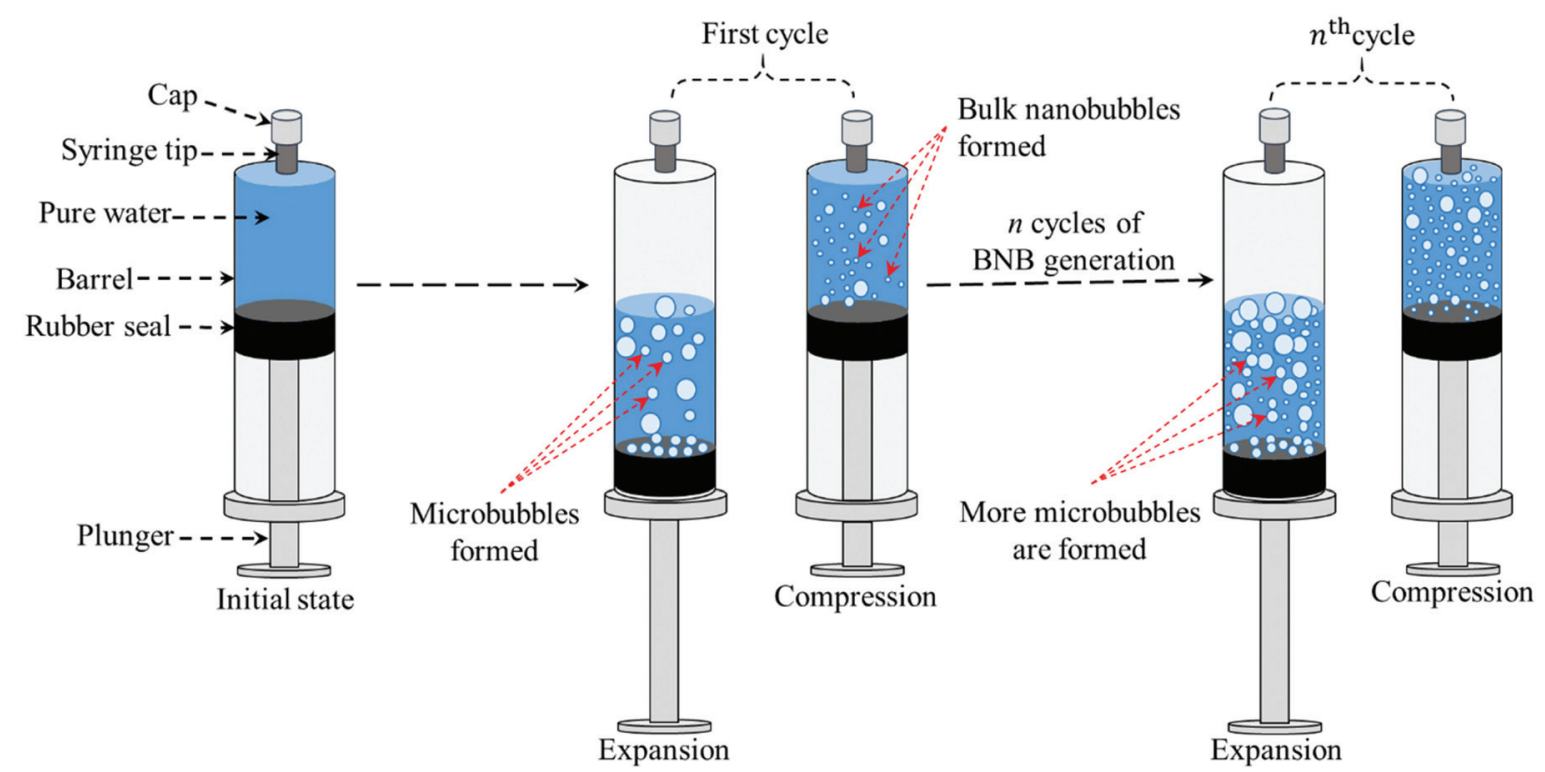

Fig. 1 Schematic representation of BNB generation process by means of successive expansion-compression cycles of pure water in a syringe.

filled syringe, which is well above the water vapour pressure of $0.023 \mathrm{~atm}$ at the same temperature. ${ }^{69}$ Hence, cavitation did not play a role in the process of BNB generation. Some limited experiments were performed at temperatures of 5, 15, 25 and $35{ }^{\circ} \mathrm{C}$ to investigate the effects of air solubility in water. As shown in Table 1, the operating vacuum pressure in the syringe was marginally below vapour pressure $(0.056 \mathrm{~atm})$ only at $35{ }^{\circ} \mathrm{C}$ where air solubility was least. Any amount of cavitation which might have occurred in this case, however, would have been insignificant and did not affect the results of the temperature experiment, as discussed further below. In conclusion, whilst the method described here has been tested solely on the basis of Henry's law effects, in practice any occurrence of cavitation can only assist the process of BNB generation. However, it is unlikely that cavitation will play any significant role even if the pressure inside the syringe falls below vapour pressure, because the time available for cavitation to take effect towards the end of the expansion stroke is very short.

The choice of syringe material and size, as well as the volume of water used for the generation of BNBs are important. After a series of tests, glass and stainless steel syringes

Table 1 Values of water vapour pressure ${ }^{67}$ and minimum operating absolute pressure inside water-filled syringe at different temperatures

\begin{tabular}{llll}
\hline $\begin{array}{l}\text { Temperature } \\
\left({ }^{\circ} \mathrm{C}\right)\end{array}$ & $\begin{array}{l}\text { Water vapour } \\
\text { pressure (atm) }\end{array}$ & $\begin{array}{l}\text { Minimum } \\
\text { operating pressure } \\
(\mathrm{atm})\end{array}$ & Cavitation \\
\hline 5 & 0.0087 & 0.048 & None \\
15 & 0.0170 & 0.048 & None \\
20 & 0.0234 & 0.048 & None \\
25 & 0.0316 & 0.048 & None \\
35 & 0.0562 & 0.048 & Insignificant
\end{tabular}

were discounted because glass syringes tended to release silica impurities, whilst stainless steel syringes generated too much friction for manual operation. Polypropylene plastic syringes with a latex-free elastomer seal are highly resistant to most solvents and chemicals ${ }^{70}$ as well as being abrasion resistant. ${ }^{71}$ They generated no significant levels of impurities and were found to be the most appropriate for manual handling. We also established that the optimum syringe size and water volume were $\sim 10 \mathrm{~mL}$ and $\sim 6 \mathrm{~mL}$, respectively, for ease of manual operation, i.e. for generating sufficient but manageable pressures, as well as providing adequate samples of BNB suspension for analysis.

It should be emphasised that successful operation of the technique relies on ensuring that the syringe is air-tight and the right level of vacuum pressure is generated; any leakage would affect the internal pressure and, hence, the performance of the BNB formation process. Prior to experiments, therefore, we used a calibrated absolute pressure sensor (MPX5700AP, 15-700 kPa, 0.2-4.7 V output; NXP Eindhoven, Netherlands) to measure the pressure online inside the syringe and ensure that the right level of vacuum was achieved consistently and precisely to ensure the reproducibility of results, as depicted in Fig. 2a. The pressure transducer was controlled by a microcontroller device (Arduino Uno Rev3 MCU) driven by a code written in LabVIEW software. ${ }^{72}$ The pressure sensor was calibrated following the manufacturer's datasheet and implementing the transducer's transfer function in the driver code written in LabVIEW, thus:

$$
V_{\text {out }}=V_{\mathrm{S}} \times(0.0012858 \times P+0.04) \pm \delta
$$

where, $V_{\text {out }}$ is the signal received from the microcontroller and detected by the pressure transducer, $V_{\mathrm{S}}$ is the voltage required by the transducer $\left(V_{\mathrm{S}}=5 \mathrm{~V}\right)$, that is the voltage the microcon- 
(a)

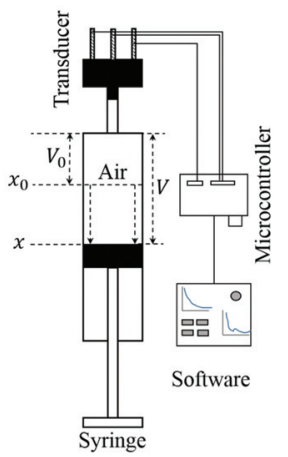

(b)

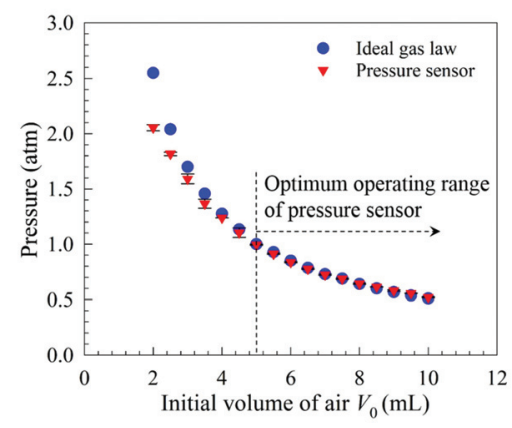

(c)

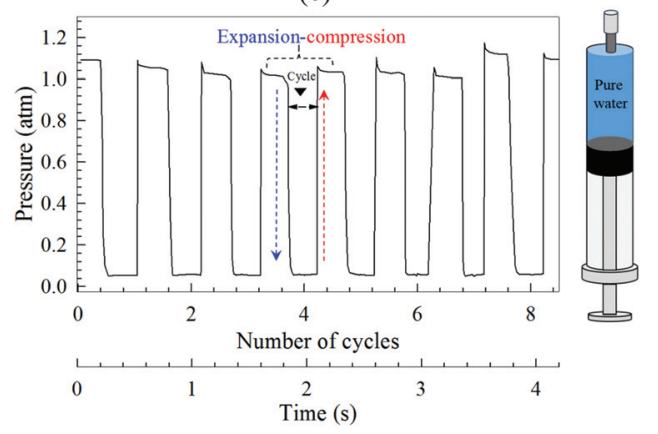

Fig. 2 Online pressure measurements inside a syringe: (a) pressure sensor setup; (b) accuracy of pressure measurement as a function of initial air volume $V_{0}$ inside air-filled syringe; (c) typical pressure variations inside water-filled syringe during generation of bulk nanobubbles.

troller provides by default to an external A/D sensor; $P$ is the pressure registered by the transducer and $\delta=0.025 V_{\text {out }}$ is the measurement error.

To test the accuracy of the pressure sensor, a PressureVolume (or $P-V$ ) curve was constructed using a 10 cc empty plastic syringe. Initially at time $t=0$, the tip of the syringe is sealed and the plunger is in its initial arbitrary position $x_{0}$ corresponding to a certain amount of air $V_{0}$ inside the syringe, which is at initial pressure $P_{0}$ equal to atmospheric pressure $\left(P_{\text {atm }}=1 \mathrm{~atm}=101.325 \mathrm{kPa}\right)$. By pulling out the plunger from $x_{0}$ to $x$, as depicted in Fig. $2 \mathrm{a}$, the volume of air inside the barrel expands from $V_{0}$ to $V$, and the internal pressure decreases according to the ideal gas law:

$$
P V=n R T
$$

where, $P, V$ and $T$ are the pressure, volume and temperature; $n$ is the number of moles and $R$ is the ideal gas constant (8.31441 $\mathrm{J} \mathrm{K}^{-1} \mathrm{~mol}^{-1}$ ). By running the experiment at constant room temperature $\left(20^{\circ} \mathrm{C}\right)$ and fixing the initial volume of air present inside the syringe $V_{0}$, it is possible to estimate the number of moles of air. Thus, the readings of the sensor could be checked against theoretical values. The most accurate experimental results with the least deviation from the ideal gas law predictions were obtained for values of $V_{0} \geq \sim 5 \mathrm{~mL}$ which delineate the optimum operating range of the pressure sensor, as shown in Fig. 2b. The actual process of generating BNBs using a water-filled syringe is schematically illustrated in Fig. 2c, showing typical cyclic pressure variations inside the syringe.

\subsection{Characterisation of bulk nanobubble suspensions}

The size distribution and the number density of BNBs were measured using a nanoparticle tracking analysis (NTA) instrument (NanoSight NS300, Malvern, UK). NTA tracks the Brownian motion of nanoparticles and is well suited for realtime analysis of polydisperse systems ranging from 10 to $2000 \mathrm{~nm}$ in size and $10^{7}$ to $10^{9}$ particles per $\mathrm{mL}$ in concentration. We recently established that, in this context, NTA is superior to dynamic light scattering (DLS) whose measurements are based on the intensity of scattered light and is consequently biased towards large particles and, hence, tends to overestimate the size of nanoparticles. ${ }^{1}$ Prior to the analysis of bulk nanobubble samples, standard suspensions of solid latex nanospheres were used to verify the accuracy and precision of the NTA system and to adjust the instrument settings accordingly. The zeta potential of the nanobubbles was measured using a Zetasizer Nano ZSP instrument (ZEN5600, Malvern, UK). These measurement techniques and their protocols as well as their theoretical foundations are discussed in more detail in our recent papers. ${ }^{1,10}$

\section{Results and discussion}

\subsection{Generation of BNBs in pure water}

The formation of BNBs by this method can probably be divided into two stages: (i) as the syringe plunger is pulled out, the water pressure reduces substantially much below atmospheric $(\sim 0.048 \mathrm{~atm})$ which results in a decrease in air solubility and creates local gas undersaturation in the form of microscopic gas packets. Since this is a closed system, the released air molecules form thousands of microbubbles which expand by virtue of Boyle's law and manifest themselves in the form of a visible milky cloud; and (ii) as the plunger is instantly released, it travels at high velocity and the compression from vacuum back to atmospheric pressure results in the conversion of microbubbles into BNBs, presumably either through microbubble collapse or gas diffusion and shrinkage. Successive cycles lead to more and more BNBs being formed. Similar observations of microbubbles being transformed into BNBs through pressure variations have been recently reported. ${ }^{23,64,65}$

The characteristics, in terms of bubble size distribution, mean bubble diameter, bubble number density and zeta potential, of the BNB suspensions generated by expansion and compression of pure water at $20^{\circ} \mathrm{C}$ are presented in Fig. 3, as a function of number of cycles. The bubble size distributions at different cycles are similar and the mean bubble diameter is approximately the same. Initially, the bubble number density rises considerably as the number of cycles increases, but the rate of increase starts to slow down as the number of cycles exceeds the first decade, and tends towards a plateau above about 25 cycles. The zeta potential of the nanobubbles, as 
(a)

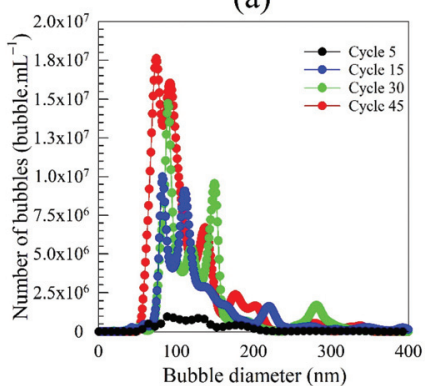

(c)

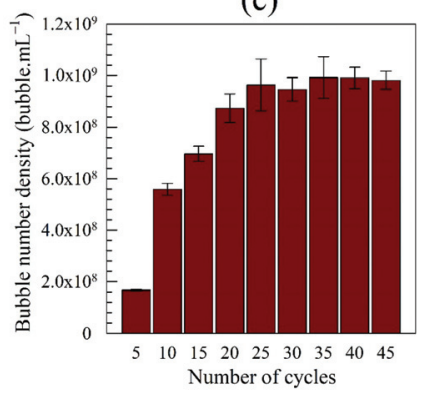

(b)

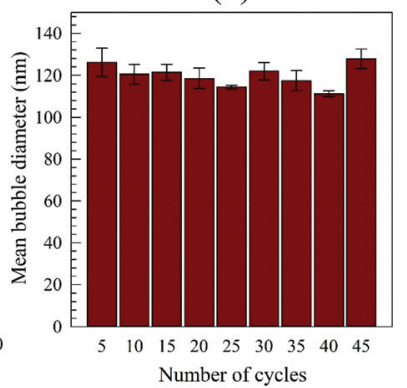

(d)

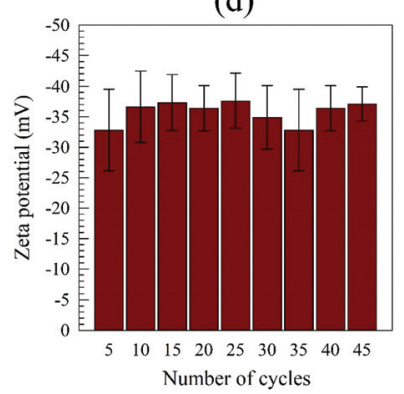

Fig. 3 Characteristics of bulk nanobubbles in pure water generated by different numbers of expansion-compression cycles: (a) bubble size distribution; (b) mean bubble diameter; (c) bubble number density; (d) zeta potential.

expected, remains unchanged within experimental error at about $-36 \mathrm{mV}$.

\subsection{Bulk nanobubbles or not nanobubbles?}

Our hypothesis is that the nano-entities produced by this expansion-compression method are nanobubbles suspended in water. We investigate a number of physical aspects and use various analytical techniques to prove this hypothesis, as follows.

3.2.1. Gradual disappearance of BNBs over time. We observed the long-term behaviour of the BNB suspensions and monitored their bubble number density, size distribution, mean bubble diameter and zeta potential. The bubble concentration slowly declined as the bubble size distribution collapsed over time, as shown in Fig. 4, indicating that the nano-
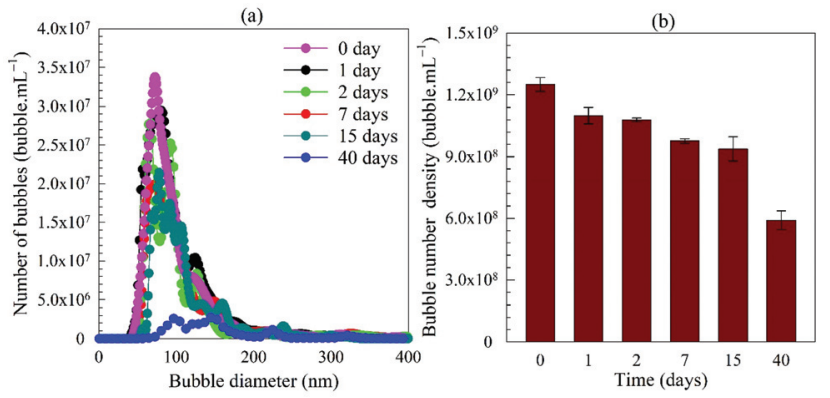

Fig. 4 Long-term evolution of BNB suspension: (a) bubble size distribution; (b) bubble number density.

entities were gradually disappearing whilst their average diameter and zeta potential retained their initial values shown in Fig. 3.

On the basis of these observations, we can safely discount the possibility of solid nanoparticles disappearing through growth in size by aggregation (constant mean diameter) and sedimentation (samples were stirred before NTA analysis). The gradual depletion of nano-entities over time, therefore, supports the hypothesis that they are gas-filled bubbles. Such a behaviour is characteristic of stable bubbles disappearing over time through no apparent breakage, coalescence or Ostwald ripening. We had recently argued that BNBs which we produced by other BNB generation methods probably disappeared by interaction with the free surface or the solid container surfaces; $;^{1,40}$ such a conjecture has been experimentally corroborated in a more recent paper. ${ }^{73}$

3.2.2. Effects of dissolved air content on generation of BNBs

3.2.2.1. Effects of water degassing. BNB generation experiments were performed, as depicted in Fig. 5a, using pure water which had been partially degassed at 15 mbar. The effects of degassing time on the formation of BNBs after 30 expansioncompression cycles are presented in Fig. 5b.

Whilst, the mean diameter remains more or less unaffected throughout, results show that the longer the water degassing time, i.e. the less the dissolved air content, the fewer the nanoentities observed per unit volume. After a degassing time of $5 \mathrm{~h}$, there is about $50 \%$ reduction in the number density of nano-entities generated compared to undegassed water. The considerable dependence of the number of nano-entities formed on the amount of dissolved air is another strong indication that they must be gas-filled nanobubbles.

3.2.2.2. Effects of air solubility. The quantity of dissolved air is affected by the solvent temperature; the lower the water temperature the higher is the air solubility. Using water at different temperatures, BNBs were generated employing different numbers of expansion-compression cycles. Results plotted in Fig. 6a show that the number of BNBs increases (a)

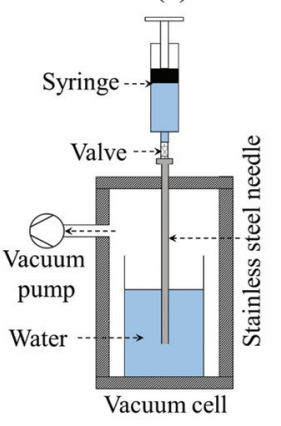

(b)

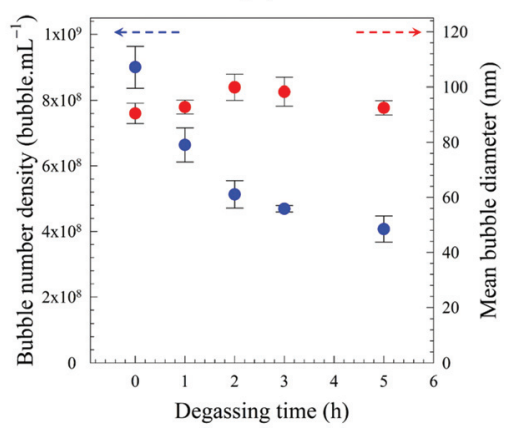

Fig. 5 Effects of water degassing on generation of bulk nanobubbles: (a) schematic representation of BNB generation process using water degassed inside vacuum cell at 15 mbar; (b) BNB measurements after 30 expansion-compression cycles. 

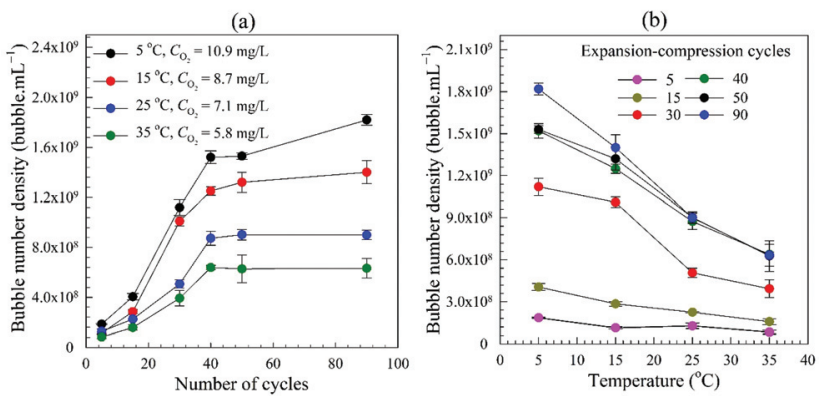

Fig. 6 Effects of air solubility on generation of bulk nanobubbles in pure water: (a) effects of number of expansion-compression cycles at constant temperature; (b) effects of temperature at constant number of expansion-compression cycles.

with the number of generation cycles. At temperatures of $25{ }^{\circ} \mathrm{C}$ and higher, the bubble number density levels off after about 40 cycles as the available released gas is depleted. However, at lower temperatures, the curve does not reach a plateau even after 90 cycles and continues to increase, albeit at a slower rate, which implies that more released air is still available in the water which requires more cycles to convert into BNBs. As expected for gas-filled bubbles, the number density is much higher at lower temperatures and declines steeply at higher temperatures, as depicted in Fig. 6b. In conclusion, these findings provide further corroboration for the assumption that the observed nano-entities are indeed gas-filled bubbles.

3.2.3. Effects of air sparging on generation of BNBs. More experiments were conducted by sparging additional air in pure water for 20 min using the setup depicted in Fig. 7, and then using such air-saturated water to produce BNBs over 30 expansion-compression cycles. After each sparging operation, a sample of the BNB suspension was withdrawn for NTA analysis and the remainder was sparged again with air for $2 \mathrm{~min}$, before being subjected to another 30 expansion-compression cycles. The process was repeated four time. Results displayed

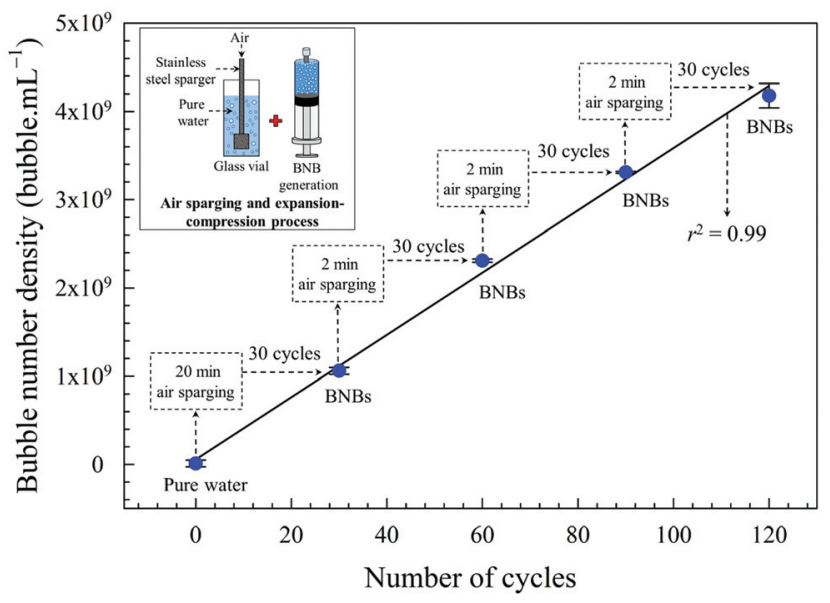

Fig. 7 Effects of repeated additional air sparging on generation of bulk nanobubbles. in Fig. 7 show that sparging enables water to be resaturated with air, thus, allowing the concentration of BNBs to increase linearly, well above the bubble concentration obtained from dissolved air only. Therefore, repeated sparging of air might provide a mechanism for producing significantly higher concentrations of BNBs, which has so far been elusive. Furthermore, these findings represent further evidence that, the number of nano-entities being significantly augmented by the supply of additional air, must be bubbles.

3.2.4. Spectroscopy analysis of BNB suspensions. Following our recent work, ${ }^{4}$ we adopted a range of chemical analytical techniques, details of which are given in ESI, $\dagger$ to show that the observed nano-entities are not due to the presence of nanoscale organic or inorganic impurities or contamination including oil nanodroplets, solid nanoparticles and supramolecules. We used Fourier transform infrared spectroscopy (FT-IR) and Raman spectroscopy analysis and compared the functional groups present in pure water and in the BNB suspensions. We also analysed pure water and BNB suspensions using gas chromatography (GC) to examine for any organic contamination. The measured spectra are presented in Fig. 8, and they show that water is the only chemical species being detected by these three different analytical techniques in the BNB suspensions.

Additionally, we used inductive coupled plasma mass spectroscopy (ICP-MS) to analyse for the presence of 20 common metal/nonmetal elements. Results summarised in Table S3 in ESI, $\uparrow$ show that the BNB suspensions contained extremely low levels of metal traces mostly similar to pure water. The concentration of $\mathrm{Si}$ in the nanobubble suspension is higher than in pure water, which suggests the existence of some syringe abrasion effects. It should be pointed out that the sample analysed by ICP-MS represents a probably worst-case scenario (a)

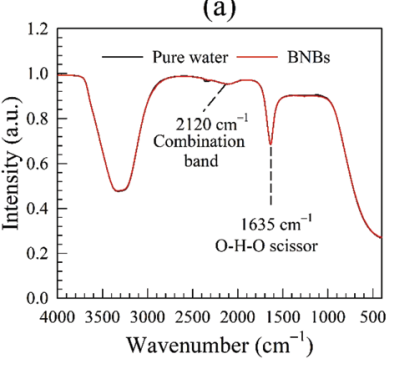

Wavenumber $\left(\mathrm{cm}^{-1}\right)$ (c)
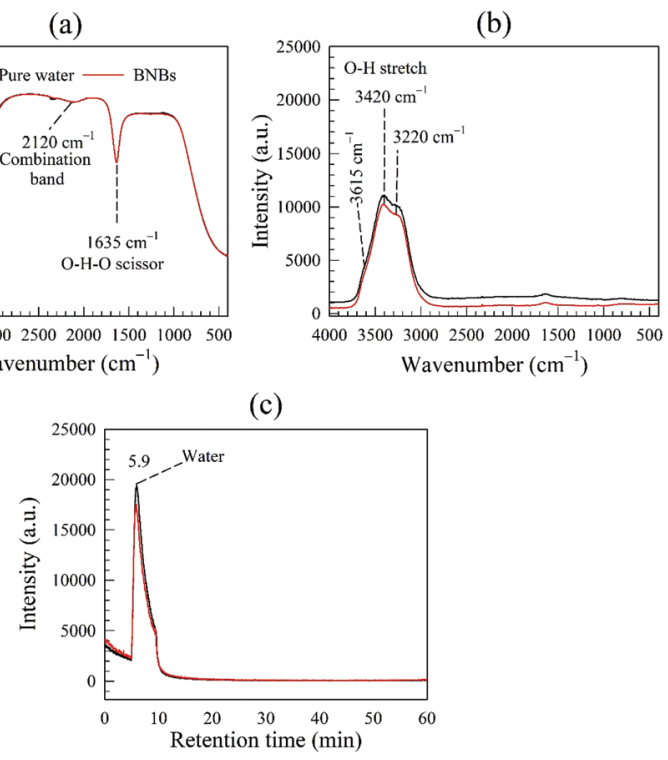

Fig. 8 Spectroscopy analysis of pure ware and bulk nanobubble suspensions: (a) FT-IR spectra; (b) Raman spectra; (c) gas chromatogram. 
where the syringe was deliberately overused ( $>400$ cycles) to assess the amount of potential impurities that can be generated and, hence, the usability of the syringe. In reality, the amount of such impurities can be controlled at much lower levels by generally restricting the number of syringe cycles to less than $\sim 200$. Nonetheless, the concentration of these impurities is still far too low and the observed nano-entities cannot, therefore, be attributed to the presence of metal/nonmetal contamination. In conclusion, these analyses combined strongly suggest that the observed nano-entities must be gasfilled bubbles.

\subsection{Properties of BNBs generated by expansion-compression method}

3.3.1. Effect of water $\mathbf{p H}$ on generation and stability of BNBs. Nanobubble suspensions were generated in pure water with the $\mathrm{pH}$ pre-adjusted in the range $2-12$ by addition of $\mathrm{HCl}$ to make acidic solutions and $\mathrm{KOH}$ to make basic solutions. Results are plotted in Fig. 9 showing the effects on bubble number density, mean bubble diameter and zeta potential. The bubble number density increases sharply as a function of pH (Fig. 9a). A large bubble number density can be achieved in acidic solutions but these BNBs which have a relatively small positive zeta potential $(\sim+10 \mathrm{mV}$; Fig. $9 \mathrm{~b})$ are relatively short-lived and generally disappear within a day or two. Their disappearance is preceded by a substantial increase in the mean bubble diameter (Fig. 9c). The zeta potential changes to negative at a pH between 4 and 5, which is expected to correspond to the isoelectric point of the solution. In these basic solutions, the bubble number density is much (up to an order

(a)
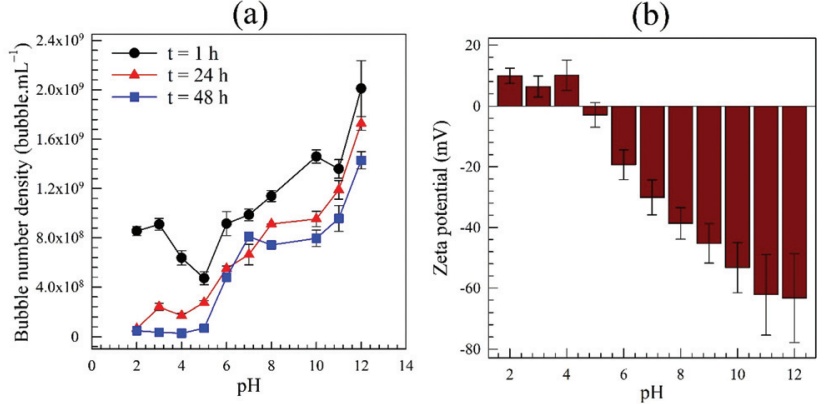

(c)

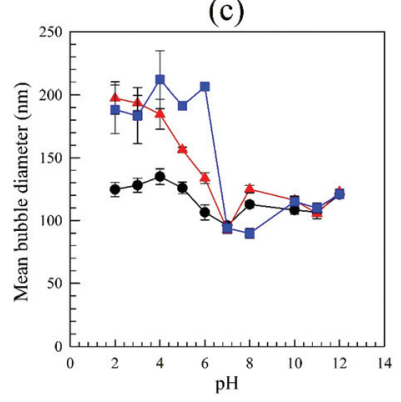

Fig. 9 Effects of pre-adjustment of water $\mathrm{pH}$ on generation and stability of bulk nanobubbles: (a) bubble number density; (b) zeta potential; (c) mean bubble diameter. of magnitude) higher and the absolute value of the zeta potential increases steadily with $\mathrm{pH}$ to reach $-63 \mathrm{mV}$ at $\mathrm{pH} 12$. Whilst the acidic BNB suspensions are short-lived, these alkaline BNBs enjoy more long-term stability and the vast majority were still in suspension after several weeks, while the mean bubble size remains approximately constant.

A plausible reason for the relatively weak stability of BNBs in acidic solutions may be advanced as follows. During production of BNBs the auto-ionisation process of water which in a neutral $\mathrm{pH}$ environment, produces an equal number of hydroxyl ions $\left(\mathrm{OH}^{-}\right)$and hydronium ions $\left(\mathrm{H}_{3} \mathrm{O}^{+}\right)$. In an acidic solution, such a process becomes heavily biased towards the production of the latter positive ions. ${ }^{74}$ Since there is a shortage in hydroxyl ions which normally attach to bubble interfaces to stabilise them, the $\mathrm{H}^{+}$ions emanating from the added $\mathrm{HCl}$ take over and attach to the bubble interfaces causing instead a positive surface potential, ${ }^{75,76}$ as shown in Fig. $9 b$.

Above the isoelectric point, the nanobubble interfaces are negatively charged (Fig. 9b). Thus, an electric double layer is expected to form around the nanobubbles, similar to that observed around solid nanoparticles. ${ }^{10}$ According to the previously postulated ion-stabilisation model, the charged nanobubble interface gives rise to an external electrostatic pressure which balances the internal Laplace pressure and, hence, no net diffusion of gas occurs at equilibrium. We previously derived expressions for these counterbalancing pressure forces from which, at equilibrium, the radius $\mathrm{R}$ of the nanobubble is given by: ${ }^{10}$

$$
R=\frac{\gamma \varepsilon}{\pi \sigma^{2}}
$$

where, $\varepsilon$ is the permittivity of the suspending medium, $\gamma$ is the surface tension and $\sigma$ is the density of surface charge which is related to the surface potential $\psi_{\mathrm{o}}$ via the Grahame equation, thus: $^{77}$

$$
\sigma=\sqrt{8 k_{\mathrm{B}} T \varepsilon \varepsilon_{0} c_{\infty}} \sin h\left(\frac{z e \psi_{0}}{2 k_{\mathrm{B}} T}\right)
$$

where, $k_{\mathrm{B}}, T, \varepsilon_{0}, c_{\infty}, z, e$ and $\psi_{0}$ are, respectively, the Boltzmann constant, temperature, permittivity of vacuum, concentration of co-ions in the bulk, the charge on ion or valence of the ionic species, elementary charge and surface potential.

It can be inferred from eqn (4) that a lower surface charge density, caused by a lower surface potential (eqn (5)), will cause the nanobubble to expand to maintain equilibrium between the inner and outer counterbalancing pressures, which probably explains why at low $\mathrm{pH}$ values the mean nanobubble diameter increases with time (Fig. 9c). Furthermore, beyond some critical nanobubble diameter, further reduction in electrostatic pressure at low $\mathrm{pH}$ may promote outward gas diffusion and, hence, disappearance of the BNBs, which could explain the sharp drop as a function of time in bubble number density observed in Fig. 9a.

Very recently, using various sets of experimental data from the literature, other authors have tested the above ion-stabilisation model. ${ }^{78}$ They showed that the excess surface charge is 
indeed responsible for the stability of bulk nanobubbles as the electrostatic effect acts as a restoring force to stabilize nanobubbles, which prevents nanobubbles in equilibrium from shrinking and growing. We previously studied the effects of adding surfactants on the stability of BNBs. The presence of such impurities tends to affect the stability of BNBs afforded by surface ions depending on the type of surfactant polarity. ${ }^{10}$ Those results further corroborate the idea of the ion-stabilisation mechanism.

3.3.2. Generation and stability of BNBs in salt solutions. The effects of pre-addition of varying concentrations of salts of different valence to pure water, namely $\mathrm{NaCl}$ (monovalent), $\mathrm{CaCl}_{2}$ (divalent) and $\mathrm{AlCl}_{3}$ (trivalent), on the generation and stability of BNBs was investigated. Results for $\mathrm{NaCl}$ and $\mathrm{CaCl}_{2}$ are presented in Fig. 10. In both cases, the presence of salt leads to a sharp drop in bubble number density which is accompanied by a considerable rise in mean bubble diameter with increasing salt concentration. In addition, the presence of either salt dramatically reduces the lifetime of BNBs.

The magnitude of the negative zeta potential decreases considerably but stays negative in the case of $\mathrm{NaCl}$, as shown in Fig. 10. In the presence of $\mathrm{CaCl}_{2}$, however, the zeta potential is positive and increases in magnitude with salt concentration (note zeta potential for concentrations greater than $50 \mathrm{mM}$

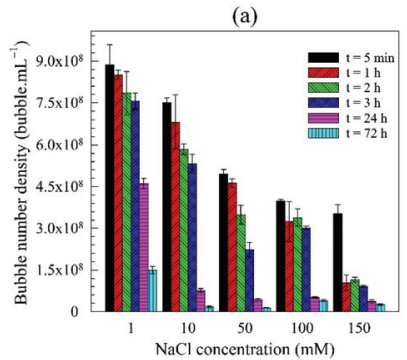

(b)

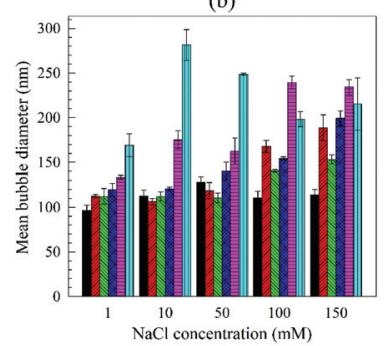

(c)

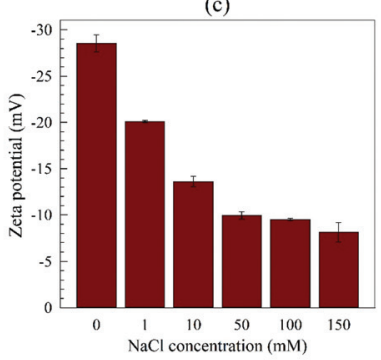

(d)

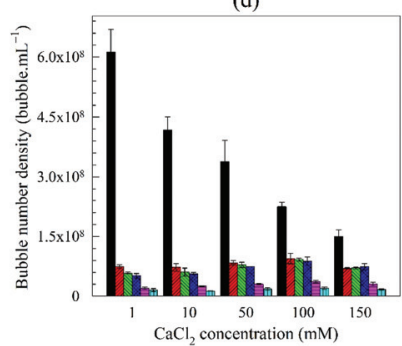

(e)

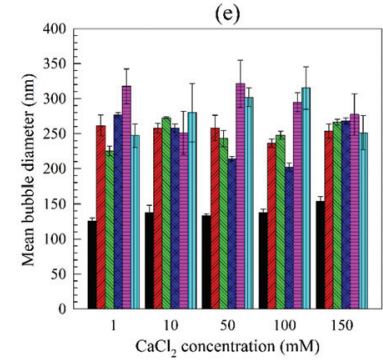

(f)

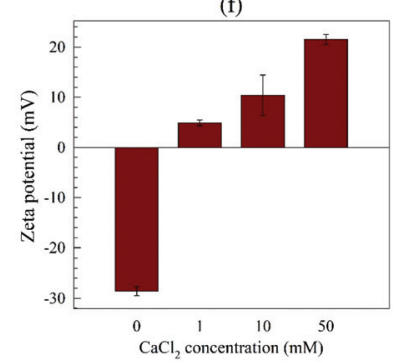

Fig. 10 Effects of pre-addition of salts on generation and stability of bulk nanobubbles: (a)-(c) $\mathrm{NaCl}$ solution; (d)-(f) $\mathrm{CaCl}_{2}$ solution. could not be measured due to the conductivity of the solution exceeding the instrument's limit). The addition of $\mathrm{AlCl}_{3}$ led to a very acidic solution $(\mathrm{pH} \sim 2.0)$ due to the formation of aqueous solution of hydrogen halide $(\mathrm{HCl})$ which made it impossible to generate BNB suspensions that are sufficiently stable for further analysis.

As pointed out above and as discussed in our recent works, ${ }^{10}$ owing to the presence of counter-ions $\left(\mathrm{OH}^{-}\right)$and coions $\left(\mathrm{H}^{+}\right)$, charged BNBs in water may form an electric double layer, the thickness of which is the Debye length $k^{-1}$ which can be estimated from:

$$
k^{-1}=\sqrt{\frac{\varepsilon \varepsilon_{0} k_{\mathrm{B}} T}{2 z_{\mathrm{i}}^{2} e^{2} c_{\infty}}}
$$

where, $\varepsilon_{0}$ is the permittivity of vacuum, $z_{\mathrm{i}}$, is the salt valence and $C_{\infty}$ is the concentration of co-ions in the bulk. In pure water, $k^{-1}=961 \mathrm{~nm}$ and, according to eqn (6), it will reduce with increasing co-ion concentration and salt valence, leading to so-called screening of the electric double layer. Due to such screening of the electric double layer, the external negative electrostatic pressure, as discussed above, would decrease leading to a pressure imbalance across the nanobubble interfaces which would then expand, possibly explaining the observed growth in mean bubble diameter.

The observed dramatic impact of the divalent salt on the zeta potential compared to the monovalent salt could, therefore, be plausibly explained by a deterioration of the electric double layer around the BNBs (i.e. $k^{-1}$ decreases) in case of the divalent salt. This is reflected in the comparatively much less stable BNBs generated in the $\mathrm{CaCl}_{2}$ solutions compared to the $\mathrm{NaCl}$ solutions (Fig. 10a and d) at any concentration; the rate of BNB disappearance being at least an order of magnitude faster in the divalent salt solution.

3.3.3. Effects of type of dissolved gas on the formation of BNBs. The effects of type of dissolved gas on the formation of BNBs in the syringe, were explored by pre-sparging air, nitrogen or Argon in pure water for $20 \mathrm{~min}$ at a controlled temperature in the range $5-35^{\circ} \mathrm{C}$. Results plotted in Fig. 11 show that increasingly more BNBs are generated with Argon than with air than with $\mathrm{N}_{2}$. The difference in bubble number density can be qualitatively explained by the differences in water solubility of these gases, i.e. Argon $>$ air $>\mathrm{N}_{2}$.

\subsection{Automation of $\mathrm{BNB}$ generation process}

Given the potentially large number of BNBs per unit volume that this expansion-compression method may be able to generate, especially with additional gas sparging (Fig. 6), we considered automating the technique to enable easy operation as well as scale-up of the BNB production process. A schematic diagram of an automated model is depicted in Fig. 12a. We used a scale which is identical to that of the manual process used in this study, i.e. a $10 \mathrm{~mL}$ syringe, to illustrate the automation of the system but the design can be readily scaled up using the same design concept. The syringe is housed inside a cylinder whilst the plunger is attached to a reciprocating pneu- 
(a)
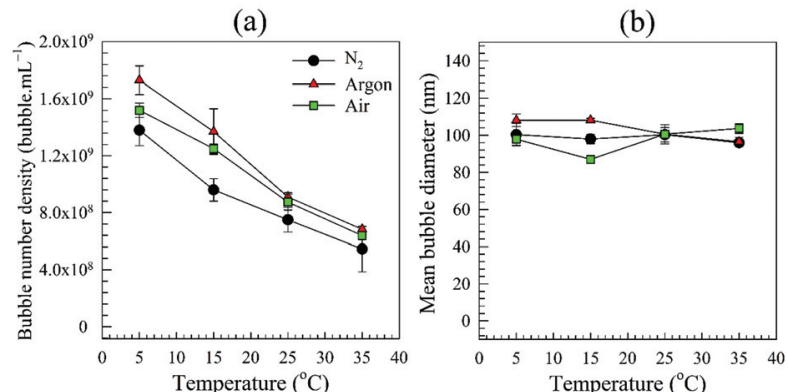

(c)

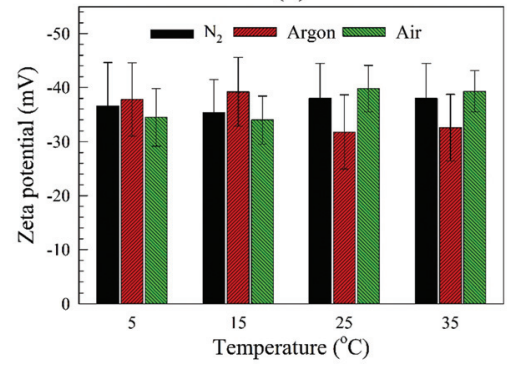

Fig. 11 Effects of type of gas on formation of BNBs: (a) bubble number density; (b) mean bubble diameter; (c) zeta potential.

(a)

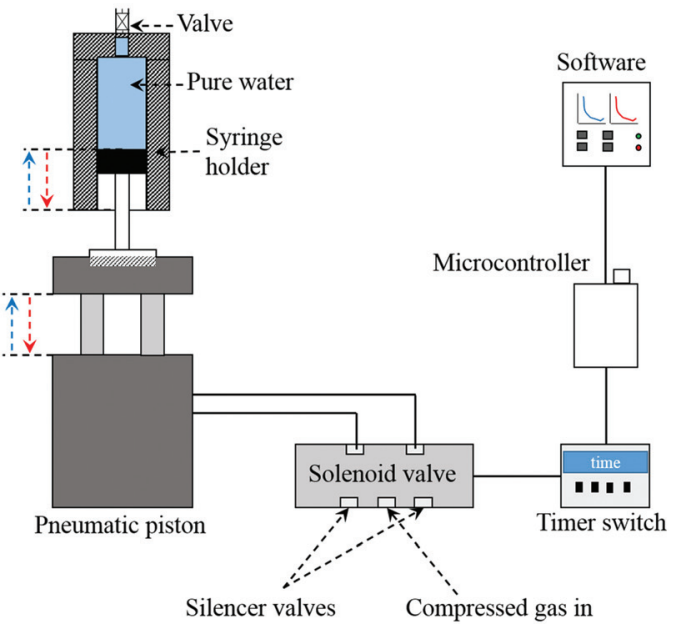

(b)

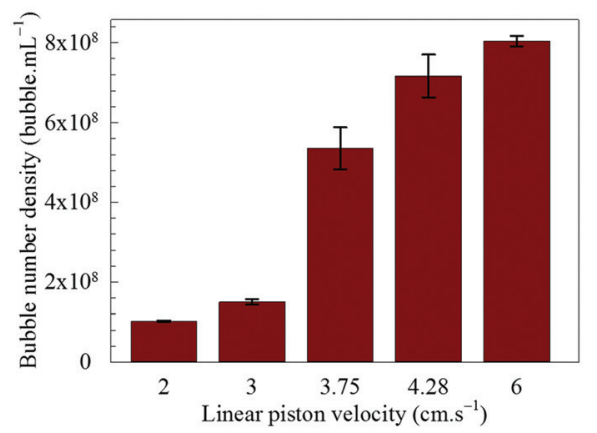

Fig. 12 Automated 1:1 scale BNB generation process: (a) schematic diagram of process setup; (b) effects of linear piston velocity at constant optimum operating gas pressure of $2.5 \mathrm{~atm}$. matic $16 \times 30$ twin-piston driven by a two-position solenoid valve operating in the pressure range $0.15-0.8 \mathrm{MPa}$ in conjunction with silencer valves, actuated by a $12 \mathrm{~V}$ trigger cycle timer delay switch.

A series of tests were conducted to establish the most efficient operating conditions for an automated 1:1 scale syringe model. First, the best operating gas pressure (2.5 bar) had to be selected within the operating range of the solenoid valve to yield the maximum bubble number density without causing mechanical damage to the syringe. Then, by keeping this gas pressure constant, the linear piston velocity was varied, as shown in Fig. 12b. The most effective piston velocity was $6 \mathrm{~cm} \mathrm{~s}^{-1}$. Higher velocities did not improve the bubble number density but caused instead increasingly more friction and mechanical stresses leading ultimately to mechanical damage of the syringe.

\section{Conclusions}

A new technique based on Henry's law's principle of vacuum degasification has been developed to generate concentrations in excess of $10^{9}$ bubble $\mathrm{mL}^{-1}$ of stable bulk nanobubbles in pure water, through successive expansion/compression strokes inside a sealed syringe. We have shown that the observed nano-entities must be gas-filled domains as: (i) they cannot be attributed, as proven by various spectroscopy analyses, to the presence of organic or inorganic impurities; (ii) they gradually disappear over time whilst their mean size remains unchanged; (iii) the amount of dissolved gas and its solubility have a direct bearing on their number density; and (iv) added sparging of gas enhances their number density.

The number of bubbles generated increases as a function of the number of expansion-compression cycles up to a point and then levels off as the available dissolved gas is depleted. However, sparging additional gas allows improved yield to be achieved.

Results on the pre-adjustment of water $\mathrm{pH}$ show that BNBs enjoy much higher stability in alkaline solutions than acidic ones. The mean size of nanobubbles increases with a decrease in $\mathrm{pH}$ whereas the bubble number density decreases. We conjecture that in pure water, due to the adsorption of $\mathrm{OH}^{-}$ions, an electric double layer similar to that observed around solid nanoparticles, forms around the negatively charged nanobubbles. The charged nanobubble interface is postulated to create an external negative electrostatic pressure which balances the internal Laplace pressure so that, at equilibrium, no net gas diffusion occurs. The disruption of this equilibrium due to a lower surface potential at low $\mathrm{pH}$, is believed to be behind the expansion and destabilisation of BNBs in acidic environments.

The presence of even small amounts of salt of any valence causes a drastic reduction in bubble number density and a sharp increase in mean bubble size, as it leads to screening of the electric double layer formed by the co-ions. As a result, the external negative electrostatic pressure decreases leading to a pressure imbalance across the interface of nanobubbles which 
then expand and grow in size; this situation is exacerbated in the case of a high salt valence.

The type of dissolved gas seems to have some effects on BNB generation. For example, more BNBs are generated with Argon than with air than with nitrogen. The difference in bubble number density can be qualitatively explained by the differences in solubility of these gases.

The proposed syringe technique has potential for large scale production of BNB suspensions. We have, therefore, successfully developed and tested an automated 1:1 scale model and have outlined the basis for process scale-up.

\section{Conflicts of interest}

There are no conflicts to declare.

\section{Acknowledgements}

This work was supported by EPSRC Grant EP/L025108/1.

\section{References}

1 N. Nirmalkar, A. W. Pacek and M. Barigou, Langmuir, 2018, 34, 10964-10973.

2 J. H. Weijs, J. R. T. Seddon and D. Lohse, ChemPhysChem, 2012, 13, 2197-2204.

3 N. Nirmalkar, A. W. Pacek and M. Barigou, Langmuir, 2019, 35, 2188-2195.

4 A. J. Jadhav and M. Barigou, Langmuir, 2020, 36, 16991708.

5 K. Ohgaki, N. Q. Khanh, Y. Joden, A. Tsuji and T. Nakagawa, Chem. Eng. Sci., 2010, 65, 1296-1300.

6 K. Ebina, K. Shi, M. Hirao, J. Hashimoto, Y. Kawato, S. Kaneshiro, T. Morimoto, K. Koizumi and H. Yoshikawa, PLoS One, 2013, 8, e65339.

7 J. N. Meegoda, S. A. Hewage and J. H. Batagoda, Environ. Eng. Sci., 2018, 35, 1216-1227.

8 P. S. Epstein and M. S. Plesset, J. Chem. Phys., 1950, 18, 1505-1509.

9 P. B. August, 2012, Big troubles over tiny bubbles, https:// www.chemistryworld.com/features/big-troubles-over-tinybubbles/5306.article, (accessed April 18, 2020).

10 N. Nirmalkar, A. W. Pacek and M. Barigou, Soft Matter, 2018, 14, 9643-9656.

11 K. Yasui, T. Tuziuti and W. Kanematsu, Ultrason. Sonochem., 2018, 48, 259-266.

12 M. Alheshibri and V. S. J. Craig, J. Phys. Chem. C, 2018, 122, 21998-22007.

13 M. Alheshibri and V. S. J. Craig, J. Colloid Interface Sci., 2019, 542, 136-143.

14 M. Alheshibri, M. Jehannin, V. A. Coleman and V. S. J. Craig, J. Colloid Interface Sci., 2019, 554, 388-395.

15 A. Häbich, W. Ducker, D. E. Dunstan and X. Zhang, J. Phys. Chem. B, 2010, 114, 6962-6967.
16 D. Rak, M. Ovadová and M. Sedlák, J. Phys. Chem. Lett., 2019, 10, 4215-4221.

17 F. Jin, J. Ye, L. Hong, H. Lam and C. Wu, J. Phys. Chem. B, 2007, 111, 2255-2261.

18 M. Sedlák and D. Rak, J. Phys. Chem. B, 2013, 117, 2495-2504.

19 V. Leroy and T. Norisuye, ChemPhysChem, 2016, 17, 27872790.

20 E. D. Michailidi, G. Bomis, A. Varoutoglou, G. Z. Kyzas, G. Mitrikas, A. Ch. Mitropoulos, E. K. Efthimiadou and E. P. Favvas, J. Colloid Interface Sci., 2020, 564, 371-380.

21 J. Qiu, Z. Zou, S. Wang, X. Wang, L. Wang, Y. Dong, H. Zhao, L. Zhang and J. Hu, ChemPhysChem, 2017, 18, 1345-1350.

22 K. Yasuda, H. Matsushima and Y. Asakura, Chem. Eng. Sci., 2019, 195, 455-461.

23 Q. Wang, H. Zhao, N. Qi, Y. Qin, X. Zhang and Y. Li, Sci. Rep., 2019, 9, 1-9.

24 A. Ushida, T. Hasegawa, T. Nakajima, H. Uchiyama and T. Narumi, Exp. Therm. Fluid Sci., 2012, 39, 54-59.

25 S. Liu, S. Oshita, Y. Makino, Q. Wang, Y. Kawagoe and T. Uchida, ACS Sustainable Chem. Eng., 2016, 4, 1347-1353.

26 S. Liu, Y. Kawagoe, Y. Makino and S. Oshita, Chem. Eng. Sci., 2013, 93, 250-256.

27 S. Calgaroto, K. Q. Wilberg and J. Rubio, Miner. Eng., 2014, 60, 33-40.

28 M. Fan, D. Tao, R. Honaker and Z. Luo, Min. Sci. Technol., 2010, 20, 1-19.

29 M. Fan, D. Tao, R. Honaker and Z. Luo, Min. Sci. Technol., 2010, 20, 159-177.

30 S. Calgaroto, A. Azevedo and J. Rubio, Miner. Eng., 2016, 89, 24-29.

31 A. Sobhy and D. Tao, Int. J. Miner. Process., 2013, 124, 109116.

32 S. H. Oh, S. H. Yoon, H. Song, J. G. Han and J.-M. Kim, Int. J. Hydrogen Energy, 2013, 38, 14849-14853.

33 S. H. Oh, J. G. Han and J.-M. Kim, Fuel, 2015, 158, 399-404.

34 J. Tian, F. Yang, H. Cui, Y. Zhou, X. Ruan and N. Gu, ACS Appl. Mater. Interfaces, 2015, 7, 26579-26584.

35 T. Yin, P. Wang, R. Zheng, B. Zheng, D. Cheng, X. Zhang and X. Shuai, Int. J. Nanomed., 2012, 7, 895-904.

36 N. Rapoport, Z. Gao and A. Kennedy, JNCI, J. Natl. Cancer Inst., 2007, 99, 1095-1106.

37 X. Fan, L. Wang, Y. Guo, Z. Tu, L. Li, H. Tong, Y. Xu, R. Li and K. Fang, PLoS One, 2015, 10, e0127419.

38 Y. Wang, X. Li, Y. Zhou, P. Huang and Y. Xu, Int. J. Pharm., 2010, 384, 148-153.

39 S. A. Peyman, J. R. McLaughlan, R. H. Abou-Saleh, G. Marston, B. R. G. Johnson, S. Freear, P. L. Coletta, A. F. Markham and S. D. Evans, Lab Chip, 2016, 16, 679-687.

40 F. Kawara, J. Inoue, M. Takenaka, N. Hoshi, A. Masuda, S. Nishiumi, H. Kutsumi, T. Azuma and T. Ohdaira, Digestion, 2014, 90, 10-17.

41 A. Ghadimkhani, W. Zhang and T. Marhaba, Chemosphere, 2016, 146, 379-384.

42 J. Zhu, H. An, M. Alheshibri, L. Liu, P. M. J. Terpstra, G. Liu and V. S. J. Craig, Langmuir, 2016, 32, 11203-11211. 
43 A. Ushida, T. Hasegawa, N. Takahashi, T. Nakajima, S. Murao, T. Narumi and H. Uchiyama, J. Surfactants Deterg., 2012, 15, 695-702.

44 A. Agarwal, W. J. Ng and Y. Liu, Chemosphere, 2011, 84, 1175-1180.

45 N. Matsuki, T. Ishikawa, S. Ichiba, N. Shiba, Y. Ujike and T. Yamaguchi, Int. J. Nanomed., 2014, 9, 4495-4505.

46 S. K. Misra, G. Ghoshal, M. R. Gartia, Z. Wu, A. K. De, M. Ye, C. R. Bromfield, E. M. Williams, K. Singh, K. V. Tangella, L. Rund, K. Schulten, L. B. Schook, P. S. Ray, E. C. Burdette and D. Pan, ACS Nano, 2015, 9, 10695-10718.

47 M. Meng, J. Gao, C. Wu, X. Zhou, X. Zang, X. Lin, H. Liu, C. Wang, H. Su, K. Liu, Y. Wang, X. Xue and J. Wu, Tumor Biol., 2016, 37, 8673-8680.

48 M. S. Khan, J. Hwang, Y. Seo, K. Shin, K. Lee, C. Park, Y. Choi, J. W. Hong and J. Choi, Artif. Cells, Nanomed., Biotechnol., 2018, 46, S318-S327.

49 M. S. Khan, J. Hwang, K. Lee, Y. Choi, K. Kim, H.-J. Koo, J. W. Hong and J. Choi, Molecules, 2018, 23, 2210-2229.

50 M. Zhou, F. Cavalieri, F. Caruso and M. Ashokkumar, ACS Macro Lett., 2012, 1, 853-856.

51 K. Kikuchi, S. Nagata, Y. Tanaka, Y. Saihara and Z. Ogumi, J. Electroanal. Chem., 2007, 600, 303-310.

52 K. Kikuchi, A. Ioka, T. Oku, Y. Tanaka, Y. Saihara and Z. Ogumi, J. Colloid Interface Sci., 2009, 329, 306-309.

53 Q. Chen, H. S. Wiedenroth, S. R. German and H. S. White, J. Am. Chem. Soc., 2015, 137, 12064-12069.

54 Q. Chen, L. Luo and H. S. White, Langmuir, 2015, 31, 45734581.

55 Q. Chen, L. Luo, H. Faraji, S. W. Feldberg and H. S. White, J. Phys. Chem. Lett., 2014, 5, 3539-3544.

56 F. Y. Ushikubo, T. Furukawa, R. Nakagawa, M. Enari, Y. Makino, Y. Kawagoe, T. Shiina and S. Oshita, Colloids Surf., A, 2010, 361, 31-37.

57 R. Etchepare, H. Oliveira, M. Nicknig, A. Azevedo and J. Rubio, Miner. Eng., 2017, 112, 19-26.

58 W. B. Zimmerman, V. Tesař and H. C. H. Bandulasena, Curr. Opin. Colloid Interface Sci., 2011, 16, 350-356.

59 A. K. A. Ahmed, C. Sun, L. Hua, Z. Zhang, Y. Zhang, W. Zhang and T. Marhaba, Chemosphere, 2018, 203, 327-335.

60 J. C. Millare and B. A. Basilia, Fluid Phase Equilib., 2019, 481, 44-54.

61 J. C. Millare and B. A. Basilia, ChemistrySelect, 2018, 3, 9268-9275.
62 J. Lombard, T. Biben and S. Merabia, Nanoscale, 2016, 8, 14870-14876.

63 E. Teirlinck, R. Xiong, T. Brans, K. Forier, J. Fraire, H. Van Acker, N. Matthijs, R. De Rycke, S. C. De Smedt, T. Coenye and K. Braeckmans, Nat. Commun., 2018, 9, 1-12.

64 J. Jin, Z. Feng, F. Yang and N. Gu, Langmuir, 2019, 35, 4238-4245.

65 J. Jin, R. Wang, J. Tang, L. Yang, Z. Feng, C. Xu, F. Yang and N. Gu, Colloids Surf., A, 2020, 589, 124430.

66 S. Ke, W. Xiao, N. Quan, Y. Dong, L. Zhang and J. Hu, Langmuir, 2019, 35, 5250-5256.

67 M. Li, L. Tonggu, X. Zhan, T. L. Mega and L. Wang, Langmuir, 2016, 32, 11111-11115.

68 A. K. Coker, in Ludwig's Applied Process Design for Chemical and Petrochemical Plants, ed. A. K. Coker, Gulf Professional Publishing, Burlington, 4th edn, 2007, pp. 103-132.

69 G. F. Hundy, A. R. Trott and T. C. Welch, in Refrigeration, Air Conditioning and Heat Pumps, ed. G. F. Hundy, A. R. Trott and T. C. Welch, Butterworth-Heinemann, 4th edn, 2016, pp. 301-312.

70 V. R. Sastri, in Plastics in Medical Devices, ed. V. R. Sastri, William Andrew Publishing, Oxford, 2nd edn, 2014, pp. 73-120.

71 A. Calhoun, in Multilayer Flexible Packaging, ed. J. R. Wagner, William Andrew Publishing, 2nd edn, 2016, pp. 35-45.

72 What is LabVIEW? - National Instruments, https:// www.ni.com/en-gb/shop/labview.html?cid=Paid_Searcha240Z0000045OsuQAE-Consideration-labview_broad\&gclid= Cj0KCQjw4dr0BRCxARIsAKUNjWR9umseVpifozT3mIdPloG mH43sq_qSun-h5LLo3GYBZMY2yccnsxYaAmQNEALw_wcB, (accessed April 15, 2020).

73 W. Kanematsu, T. Tuziuti and K. Yasui, Chem. Eng. Sci., 2020, 219, 115594.

74 M. Moqadam, A. Lervik, E. Riccardi, V. Venkatraman, B. K. Alsberg and T. S. van Erp, Proc. Natl. Acad. Sci. U. S. A., 2018, 115, E4569-E4576.

75 A. Phukan, K. S. Goswami and P. J. Bhuyan, Phys. Plasmas, 2014, 21, 084504.

76 G. Liang, W. Chen, A. V. Nguyen and T. A. H. Nguyen, J. Colloid Interface Sci., 2018, 517, 230-238.

77 G. H. Findenegg, Ber. Bunsenges. Phys. Chem., 1986, 90, 1241-1242.

78 H. Zhang, Z. Guo and X. Zhang, Soft Matter, 2020, 16, 5470-5477. 